\title{
Mesiodistal tooth size in non-syndromic unilateral cleft lip and palate patients: a meta-analysis
}

\author{
Gregory S. Antonarakis • Kleopatra Tsiouli • Panagiotis Christou
}

Received: 13 September 2011 / Accepted: 1 August 2012 / Published online: 23 August 2012

(C) Springer-Verlag 2012

\begin{abstract}
Objectives To evaluate, using meta-analysis methodology, mesiodistal tooth dimensions in non-syndromic unilateral cleft lip and palate (CLP) patients.

Materials and methods A literature search was conducted using PubMed, Medline, Google Scholar Beta, EMBASE Excerpta Medica, CINAHL, Web of Science, and the Cochrane Collaboration, identifying English and nonEnglish articles reporting on mesiodistal tooth dimensions on the cleft and non-cleft side of non-syndromic unilateral CLP patients. Additional studies were identified by searching reference lists of articles consulted. Only studies with a suitable control group were included. Two examiners independently performed the literature search and data extraction. Using meta-analysis software, data extracted from each selected study were statistically combined using the fixedeffects model. Weighted mean differences, $95 \%$ confidence intervals, and heterogeneity were calculated for each measurement.
\end{abstract}

G. S. Antonarakis and K. Tsiouli contributed equally.

\section{G. S. Antonarakis $(\bowtie)$}

Department of Orthodontics, Dental School, University of Geneva,

19 , rue Barthelemmy-Menn,

1205 Geneva, Switzerland

e-mail: Gregory.Antonarakis@unige.ch

K. Tsiouli

Private Practice,

Athens, Greece

P. Christou

Department of Orthodontics, Dental School,

National and Kapodistrian University of Athens,

Athens, Greece
Results Four articles fulfilling the inclusion criteria were located and included in the meta-analysis. Maxillary incisors and first molars were found to be significantly larger on the non-cleft side while mandibular incisors and premolars were larger on the cleft side, in nonsyndromic unilateral CLP patients. On the cleft side, maxillary premolars and second molars were larger in cleft than control patients while incisors were smaller, whereas all mandibular teeth were larger in cleft patients. On the non-cleft side, all maxillary teeth except for the central incisors were larger in the cleft than control patients, while all mandibular teeth were larger in the cleft patients except for lateral incisors.

Conclusions Non-syndromic unilateral CLP patients tend to have larger posterior but smaller anterior teeth compared with the general population. Comparing sides, unilateral CLP patients tend to have smaller maxillary but larger mandibular teeth on the cleft than on the non-cleft side.

Clinical relevance Given that obtaining a stable, functional, and esthetic occlusion requires a thorough evaluation of tooth size, knowledge about trends in tooth size variations in CLP patients can help with dental and orthodontic treatment planning.

Keywords Cleft lip and palate $\cdot$ Tooth size $\cdot$ Orthodontics . Meta-analysis

\section{Introduction}

To obtain a stable, functional, and esthetic occlusion, dental and orthodontic treatment plans need to take into account tooth size [1]. Obtaining ideal intermaxillary relationships 
and occlusion, as well as post-treatment stability, requires proper alignment of the teeth in both dental arches, with adequate overjet and overbite. Tooth size is something that clinicians need to adequately consider during treatment planning, as discrepancies in tooth size between the arches can prevent the proper alignment of teeth, and thus recording these discrepancies before treatment is necessary to obtain ideal occlusion after treatment [1]. The latter is of particular challenge in cleft lip and palate (CLP) patients.

Tooth size is multifactorial in nature, determined both by genetic and environmental factors [2]. Genetics are thought to play an important role in dictating tooth size [3, 4], implying that, to a certain extent, tooth dimensions are dependent on the genetic make-up of every individual. At the same time, however, etiological factors in the prenatal and postnatal periods have been reported to cause anomalies in tooth dimensions and morphology [5]. In a study on monozygotic and dizygotic twins, Lundström [6] proposed that genetic factors affected tooth size at least as much as did environmental factors. Normal variation in tooth size is thus the result of both genetic and environmental controls [7, 8].

Permanent tooth crown size and shape are partial indicators of a person's morbidity, general well-being, and growth capacity $[9,10]$. It is thus hypothesized that a compromised growth potential, such as seen in CLP individuals, might be reflected in smaller teeth [7]. The dentition is said to provide valuable retrospective information on the development of individuals with CLP, allowing a means of assessing growth at various ages, by appraising teeth that mineralized at specific intervals during growth [11]. The completed crown size of teeth is formed during a span of several years, and once formed, the crowns are readily measured and are not altered except by tooth wear and disease [7].

Dental abnormalities, such as disturbances in number, size, shape, and timing of formation, demonstrate increased frequency in children affected with CLP than in the general population [7, 11-22]. Abnormal tooth size and morphology are two of the most prevalent abnormalities observed [14].

One of the best general indicators of developmental instability is the asymmetry of bilaterally paired structures. It is assumed that the same genetic and general environmental factors control development on the two sides of the body, so that the extent to which sides differ is a measure of lack of precision of this developmental control [23]. As a consequence, apart from differences in tooth size between CLP patients and the general population, differences may also be present in unilateral CLP individuals between the side with the cleft and the side without. Even in a general population, dental asymmetry is usually present, but the differences between the left and right sides are small and may be of no statistical or clinical significance [24]. Garn et al. [25] on the other hand conclude that the same teeth on the left and right sides are of different sizes, especially the lateral incisors, first premolars, and first molars, in both upper and lower jaws.

Having recognized that, in order to achieve proper occlusion and stability, tooth dimensions of both arches must be considered [1], it was decided to concentrate on mesiodistal (MD) tooth dimensions for the purpose of the present study. Many studies have compared discrepancies in tooth size. The largest MD dimension has been reported to be the most reliable and appropriate measurement for representing the size of the crown [26]. Concerning CLP patients, the literature reveals that, although there has been a substantial amount of research into variations in tooth morphology, there are marked variations in the published results [16] as regards tooth size. Studies often tend to have small sample sizes, which can lead to bias as regards interpretation of results [16].

The aim of the present study was to systematically review the literature concerning MD tooth dimensions in nonsyndromic unilateral CLP patients and using meta-analysis methodology to specifically evaluate (1) MD tooth dimensions in non-syndromic unilateral CLP patients versus a general population and (2) MD tooth dimensions between the cleft and the non-cleft side in non-syndromic unilateral CLP patients. Our hypothesis was that differences would exist in the maxillary dentition between non-syndromic unilateral CLP patients and the general population on the cleft side, and between the cleft and the non-cleft side in non-syndromic unilateral CLP patients. No differences were expected in the mandibular dentition.

\section{Materials and methods}

The present meta-analysis was carried out following the PRISMA (preferred reporting items for systematic reviews and meta-analyses) statement for reporting systematic reviews and meta-analyses of studies that evaluate healthcare interventions [27] as best applicable to the present meta-analysis.

\section{Literature search}

A literature search was conducted to identify articles reporting on MD tooth dimensions on the cleft and non-cleft side of non-syndromic unilateral CLP patients in comparison to a general population, using the following databases: PubMed, Medline, Google Scholar Beta, EMBASE Excerpta Medica, CINAHL, Web of Science, and the Cochrane Collaboration. The last search was performed in the fourth week of January 2011.

The following search terms were used: cleft lip and/or cleft palate; tooth size or tooth dimensions. No language, publication date, or publication status restrictions were 
imposed. The search was expanded by searching reference lists of articles consulted, to identify other relevant articles.

Articles were selected for inclusion based on the following inclusion criteria:

- Human studies

- Examination of non-syndromic unilateral cleft lip and palate patients

- Sample of at least ten patients

- MD tooth measurements present

- Permanent teeth only measured

- Divided cleft side and non-cleft side

- Included a suitable control (non-cleft) group

- Number of subjects (cleft and control groups) mentioned

- Descriptive statistics with mean and standard deviation for both cleft and control groups mentioned

Articles were excluded based on the following exclusion criteria:

- Syndromic cleft lip and palate patients

- Bilateral cleft lip and palate patients

- Sample of less than ten patients, or not mentioned

- Deciduous teeth measured

- Combined data for the cleft and non-cleft sides

- No suitable control (non-cleft) group

- Insufficient statistics

In the case of more than one publication about the same patient group, the most informative and relevant article was included. Two examiners independently performed the literature search in order to maximize the number of studies retrieved. All selected articles were assessed by the two examiners for the predefined inclusion criteria, and

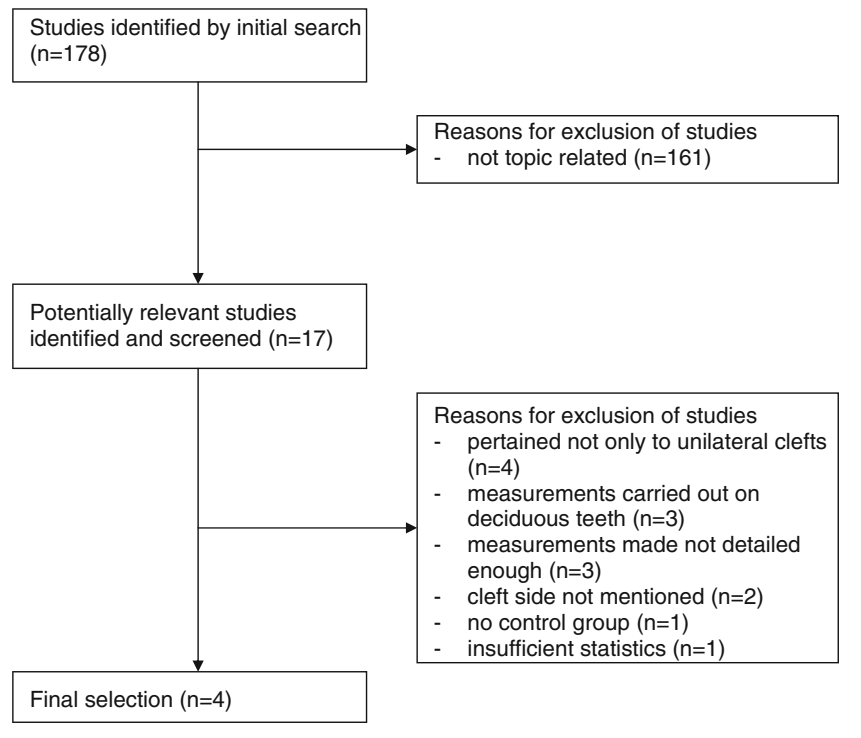

Fig. 1 Flow diagram summarizing literature search and article selection

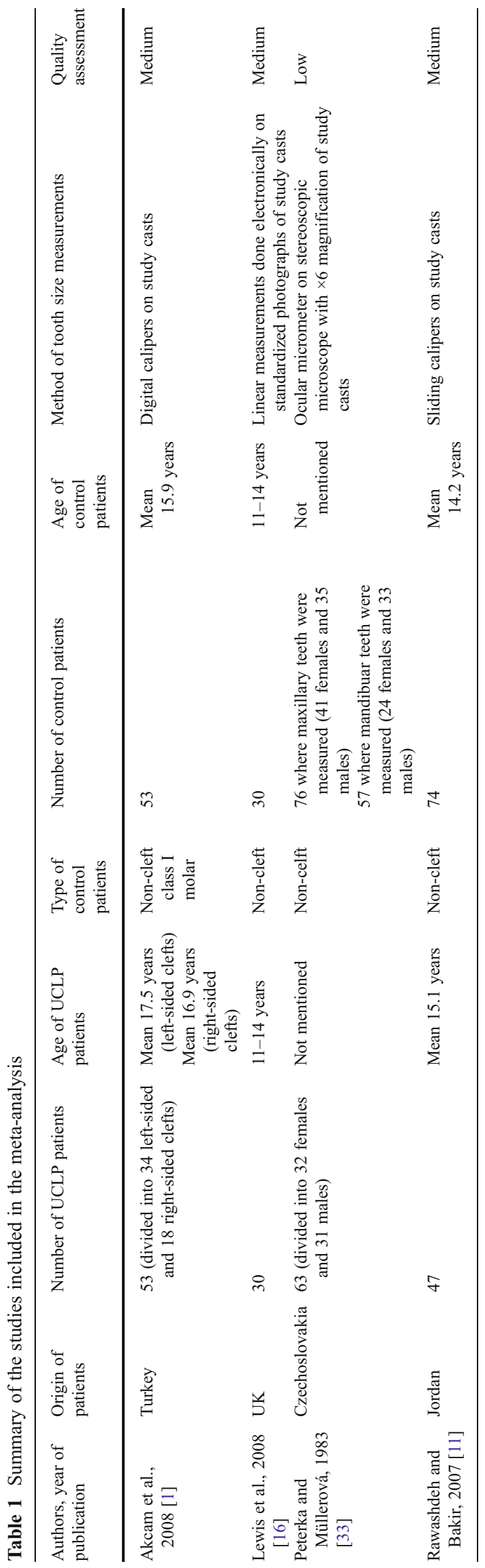


Fig. 2 Forest plots representing MD tooth dimensions for the maxillary arch on the cleft side and non-cleft side of unilateral CLP patients. The studies are listed in chronological order and refer to the studies summarized in Table 1. Shown for every study is the sample size $(N)$, weighted mean difference (WMD) between the cleft and non-cleft side, as well as the $95 \%$ confidence interval (95\% CI) for each measurement. The diamonds represent the overall WMD and $95 \%$ CI. $I^{2}$ values and $\chi^{2}\left(\mathrm{Chi}^{2}\right)$ values (for heterogeneity) and $p$ values (for statistical significance) are shown below each forest plot

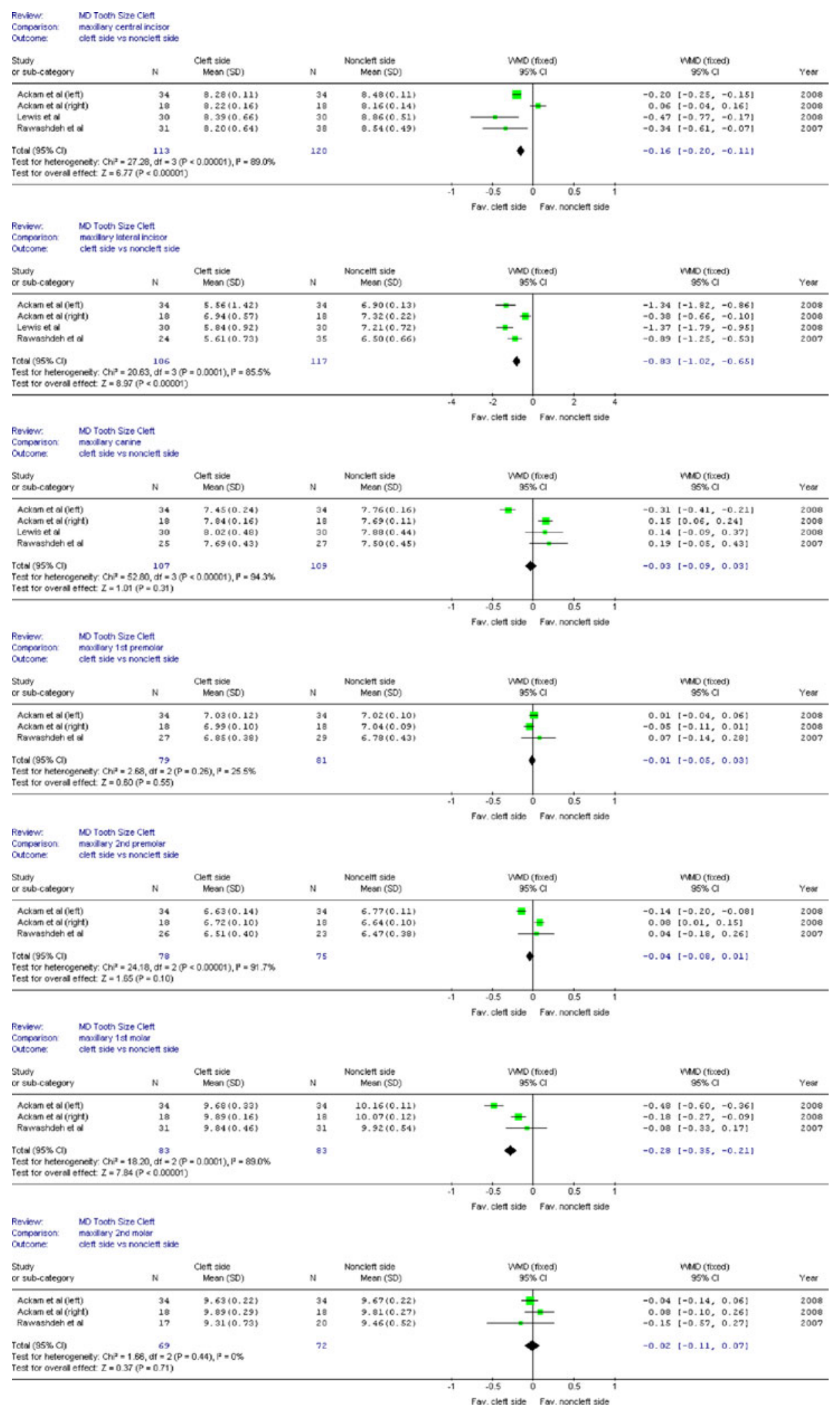

eligibility assessment was performed independently by the two examiners. Any disagreement was resolved by discussion and consensus.
A quality assessment of the included studies was carried out in order to evaluate their methodological soundness. This was performed using the methods 
Fig. 3 Forest plots representing MD tooth dimensions for the mandibular arch on the cleft side and non-cleft side of unilateral CLP patients. The studies are listed in chronological order and refer to the studies summarized in Table 1. Shown for every study is the sample size $(N)$, weighted mean difference (WMD) between the cleft and non-cleft side, as well as the $95 \%$ confidence interval (95\% CI) for each measurement. The diamonds represent the overall WMD and $95 \% \mathrm{CI}$. $I^{2}$ values and $\chi^{2}\left(\mathrm{Chi}^{2}\right)$ values (for heterogeneity) and $p$ values (for statistical significance) are shown below each forest plot

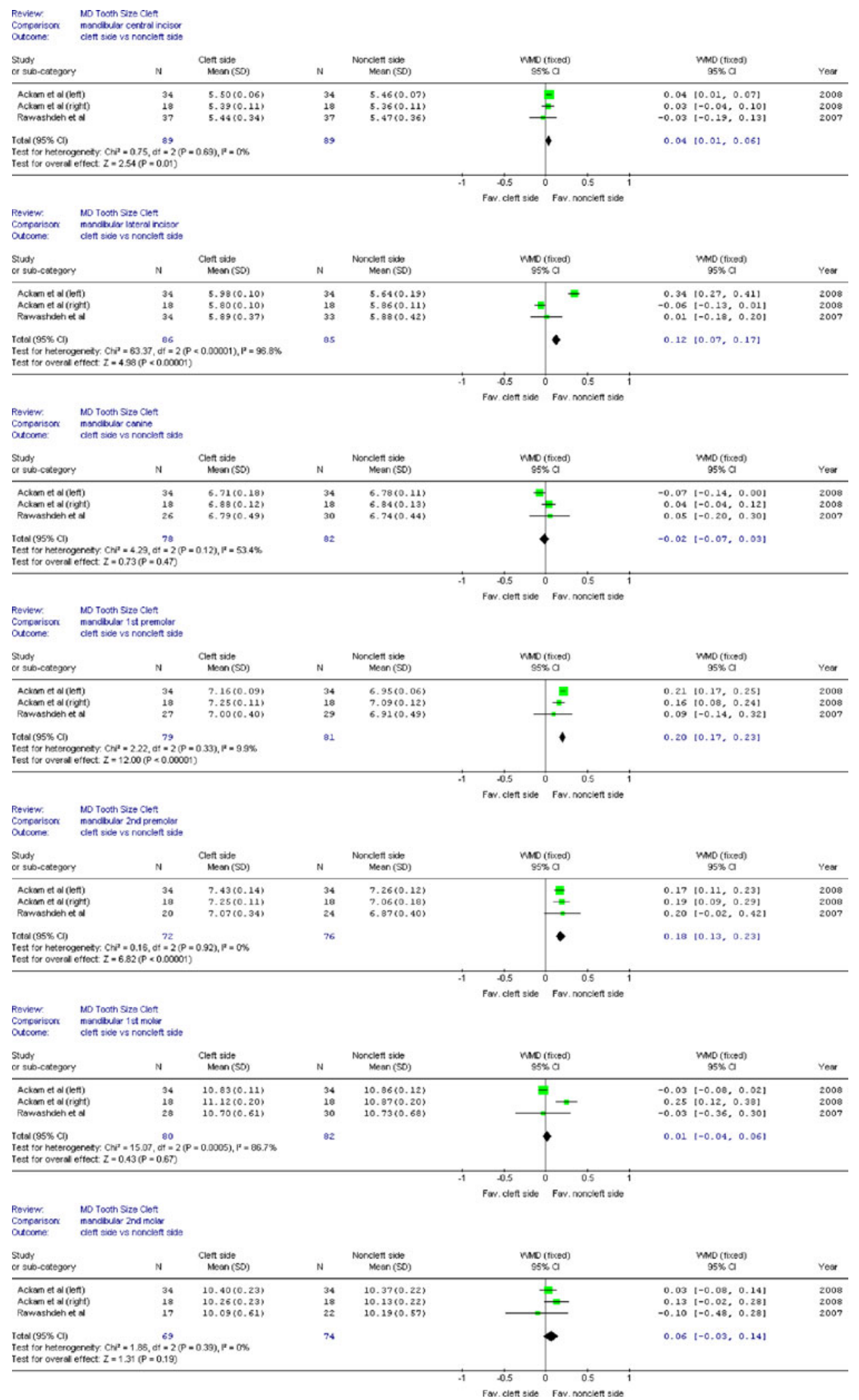

described by Jadad et al. [28] and Petrén et al. [29]. The article quality was judged as low, medium, or high based on characteristics including study design, sample size, selection description, valid methods, method of error analysis, blinding in measurements, and adequate statistics. The assessment was performed by the two examiners, and in case of discrepancy, a consensus decision was taken following discussion. 


\section{Data extraction and analysis}

Information was extracted from each included study on: number of cleft patients; type and side of unilateral cleft; number of control patients; type of control patients; and means and standard deviations for MD tooth dimensions on the cleft side, non-cleft side, and control teeth of all teeth measured (central incisors to second molars for each side). The primary outcome measure was the mean difference in MD tooth dimensions.

The data were subsequently entered into the metaanalysis software of the Cochrane Collaboration (RevMan 4.2.8, released 8 July 2005). Using the fixed-effects model, forest plots were drawn and significance tests carried out (calculating $P$ values) comparing MD tooth sizes of the cleft versus the non-cleft side in the CLP patients, the cleft side in CLP patients versus the same side in the control patients, and the non-cleft side in CLP patients versus the same side in the control patients. The results of individual studies were statistically combined into a single measure, a feature distinctive of meta-analysis. A weighted mean difference was calculated, taking into account sample size, as well as $95 \%$ confidence intervals for each measurement.

Heterogeneity tests were also performed. If confidence intervals for the results of individual studies (depicted graphically using horizontal lines) have poor overlap, this generally indicates the presence of statistical heterogeneity. More formally, statistical tests for heterogeneity are available. These include $\chi^{2}$ tests as well as calculation of $I^{2}$. The $\chi^{2}$ test is based on the sum of the squared difference between the treatment effect of each individual trial and overall treatment effect, weighted by the inverse of the variance in each trial. It assesses whether observed differences in results are compatible with chance alone. A low $P$ value (or a large $\chi^{2}$ statistic relative to its degree of freedom) provides evidence of heterogeneity of treatment effects (variation in effect estimates beyond chance) [30].

A more useful statistic for quantifying inconsistency, and present in the forest plots, is $I^{2}=[(Q-d f) / Q] \times 100 \%$, where $Q$ is the $\chi^{2}$ statistic and $d f$ is its degree of freedom $[31,32]$. This describes the percentage of the variability in effect estimates that is due to heterogeneity rather than sampling error (chance). A value greater than $50 \%$ may be considered substantial heterogeneity [30].

\section{Results}

Results of literature search

The search strategy resulted in a total of 178 articles. The articles were narrowed down to 17 after the initial exclusion based on the content of the abstract. These 17 articles were read and examined in detail, and following consideration of the inclusion criteria, a total of four articles [1, 11, 16, 33] were identified for inclusion in the meta-analysis. Figure 1 provides an overview of the literature search and article selection in the form of a flow diagram. Table 1 gives a summary of the studies included in the meta-analysis. Using the quality analysis, three of the studies were classified as having medium quality $[1,11,16]$ while one was classified as having low quality [33].

\section{Cleft versus non-cleft side (Figs. 2 and 3)}

When comparing MD tooth sizes of the cleft side versus the non-cleft side in the non-syndromic unilateral CLP patients, one can see several differences. In the maxillary dentition, the central incisors, lateral incisors, and first molars are significantly larger on the non-cleft than on the cleft side. All of these results however show low homogeneity. In the mandibular dentition, the central and lateral incisors, and first and second premolars are larger on the cleft than on the non-cleft side. All of these results show high homogeneity except for the lateral incisors.

\section{Cleft side versus control (Figs. 4 and 5)}

When comparing MD tooth sizes of the cleft side in the nonsyndromic unilateral CLP patients versus the same side in the control patients, one can see the following differences. Maxillary second molars and first and second premolars of the cleft side in non-syndromic unilateral CLP patients are larger than in control patients, while the maxillary central and lateral incisors are smaller. As regards the mandibular dentition, all of the mandibular teeth are larger on the cleft side in non-syndromic unilateral CLP patients than in control patients. These results present low homogeneity.

Non-cleft side versus control (Figs. 6 and 7)

When comparing MD tooth sizes of the non-cleft side in the non-syndromic unilateral CLP patients versus the same side in the control patients, a similar trend is seen as for the cleft side versus the control patients. In the maxillary arch, all teeth except for the central incisors are larger in the nonsyndromic unilateral CLP patients than in the control patients but with a low homogeneity seen in the results. The central incisors are larger in the control patients, again with a low homogeneity. In the mandibular arch, all teeth are larger in the non-syndromic unilateral CLP patients than in the control patients, except for the mandibular lateral incisors which are larger in the control patients. Low homogeneity is again seen here apart from the results for the mandibular first molars. 
Fig. 4 Forest plots representing MD tooth dimensions for the maxillary arch on the cleft side in unilateral CLP patients and the same side in control patients. The studies are listed in chronological order and refer to the studies summarized in Table 1 . Shown for every study is the sample size $(N)$, weighted mean difference (WMD)

between the cleft and non-cleft side, as well as the $95 \%$ confidence interval $(95 \% \mathrm{CI})$ for each measurement. The diamonds represent the overall WMD and $95 \%$ CI. $I^{2}$ values and $\chi^{2}\left(\mathrm{Chi}^{2}\right)$ values (for heterogeneity) and $p$ values (for statistical significance) are shown below each forest plot
Review: Mo Tooth size clen

Comperison: mondbulur central inctaor

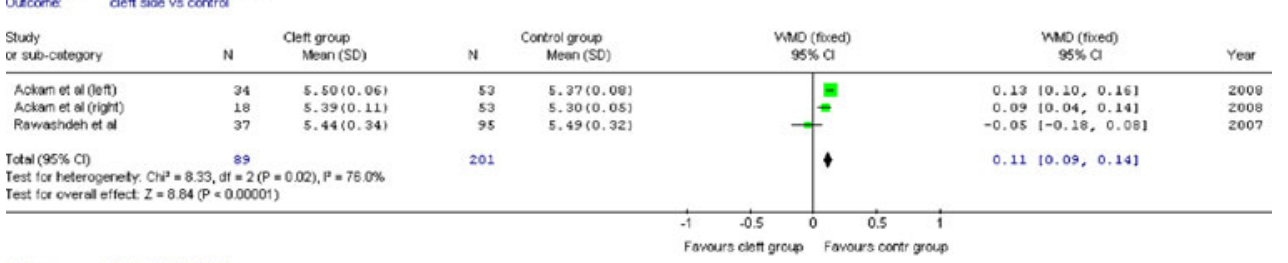

Review: MD Tooth 5 Lize Clent

Comparison mandbular lateral incisos

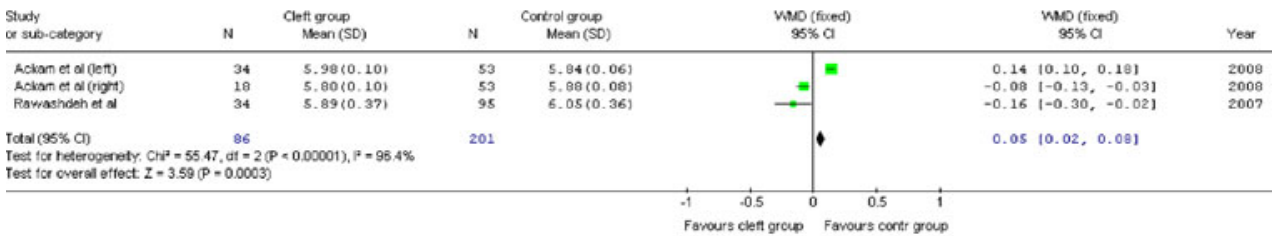

Reviews: MD Tooth Size Cleft

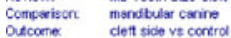

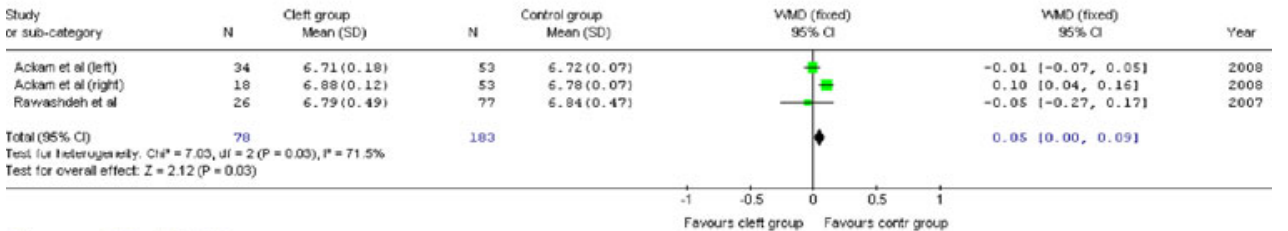

Review. MD Tooth Size Cleft
Comparieon

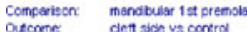

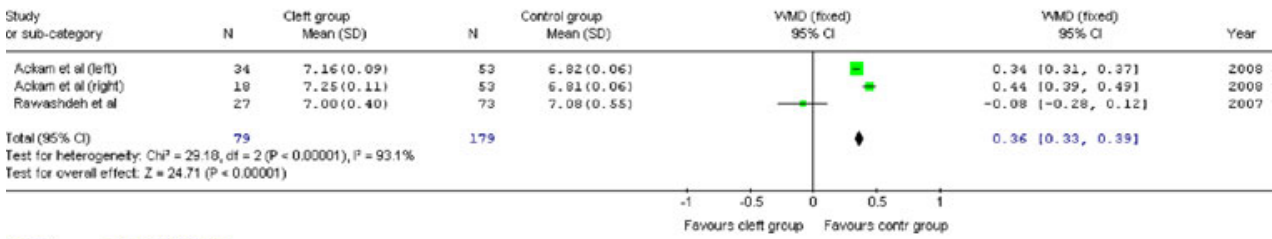

Review: MD Tooth Size Clen

Comporison mandbular and prem

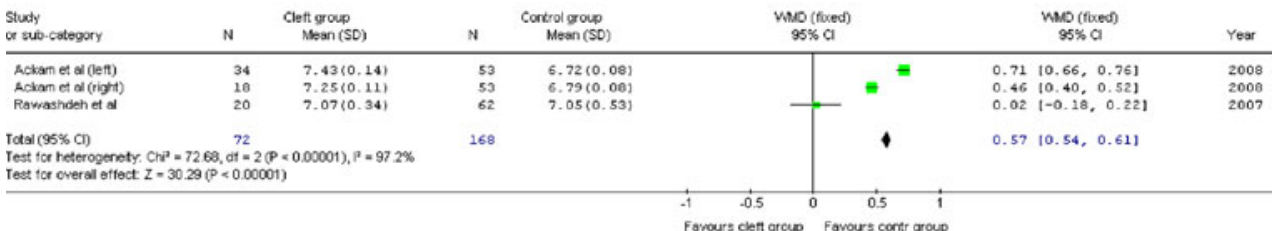

$\begin{array}{ll}\text { Review: } & \text { MO Tooth Size Cleft } \\ \text { Comperison } & \text { manobular } 1 \text { st molar }\end{array}$

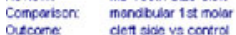

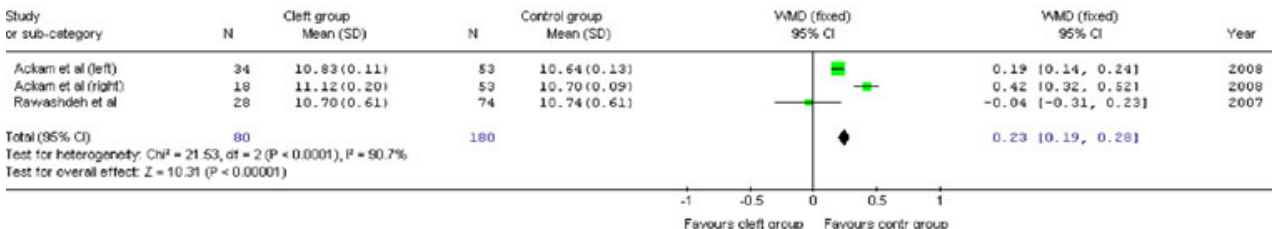

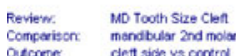

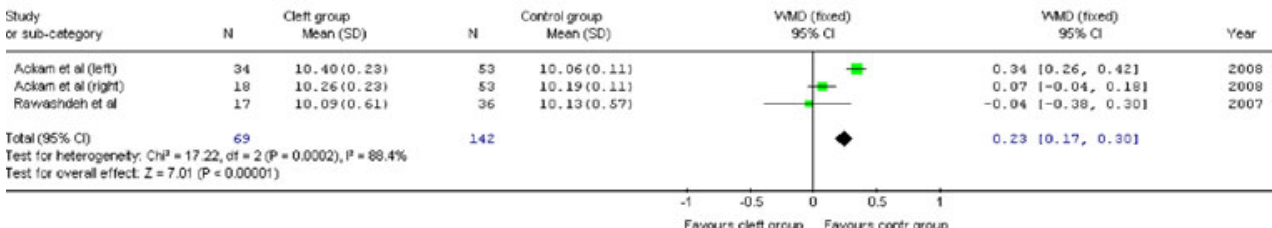


Fig. 5 Forest plots representing MD tooth dimensions for the mandibular arch on the cleft side in unilateral CLP patients and the same side in control patients. The studies are listed in chronological order and refer to the studies summarized in Table 1 . Shown for every study is the sample size $(N)$, weighted mean difference (WMD)

between the cleft and non-cleft side, as well as the $95 \%$ confidence interval $(95 \% \mathrm{CI})$ for each measurement. The diamonds represent the overall WMD and $95 \%$ CI. $I^{2}$ values and $\chi^{2}\left(\mathrm{Chi}^{2}\right)$ values (for heterogeneity) and $p$ values (for statistical significance) are shown below each forest plot

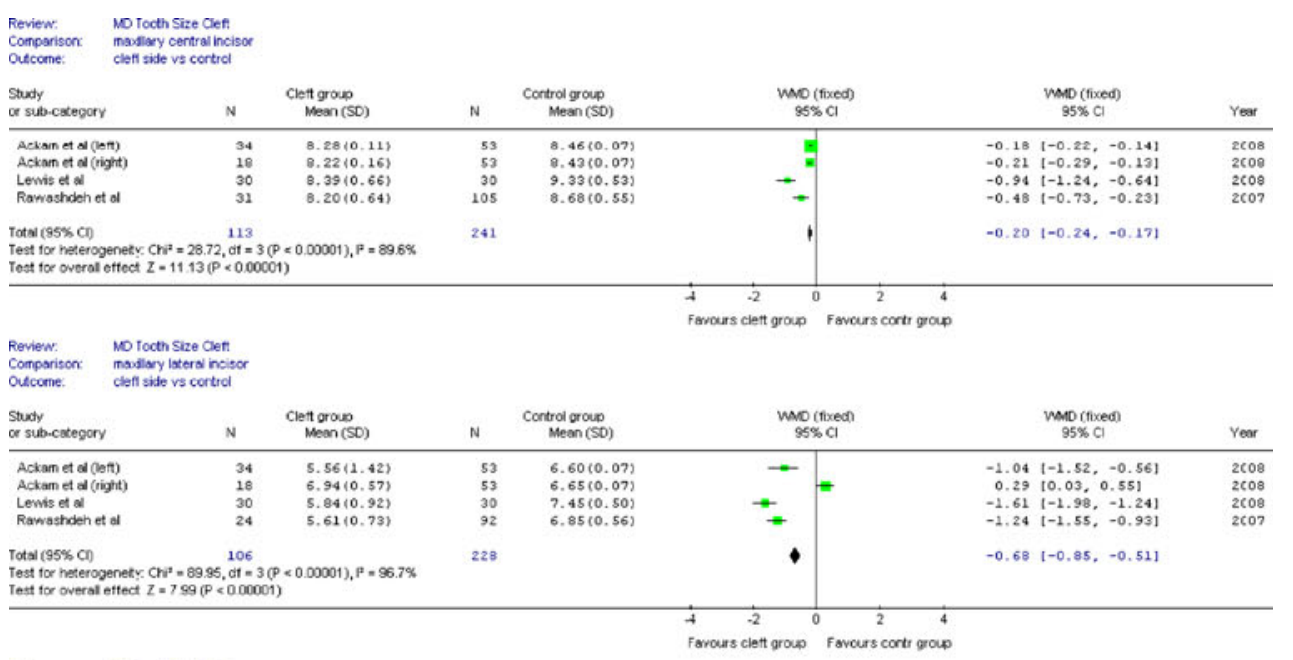

Review: Mo locth Size Clen:
Combarison: madlay canine

outcome: clefl side vs cortt

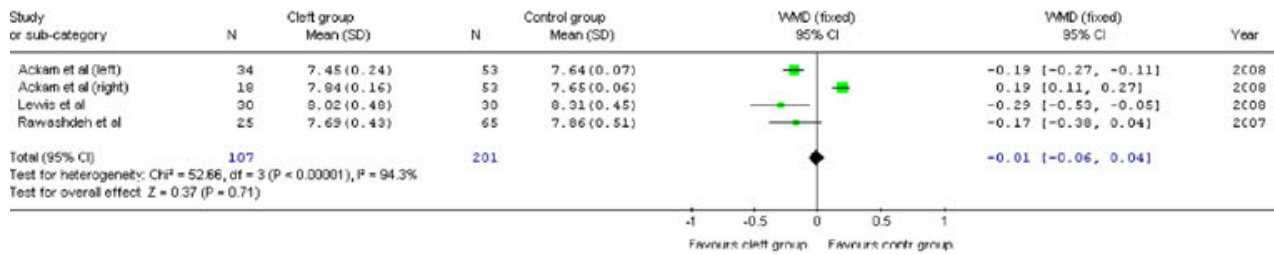

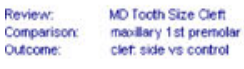

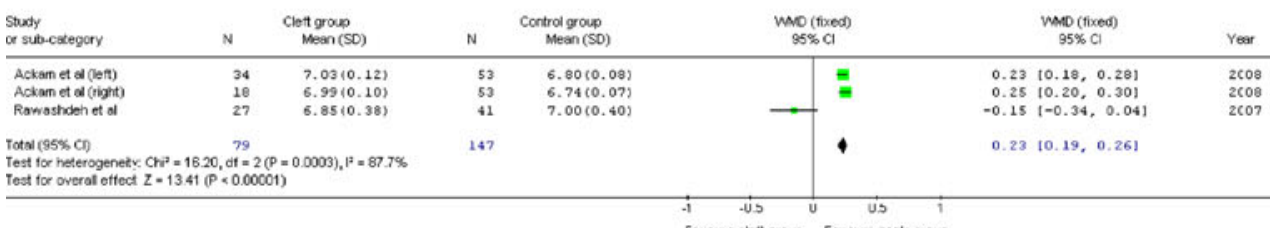

Review: Mo Tocth Size Cet:

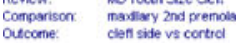

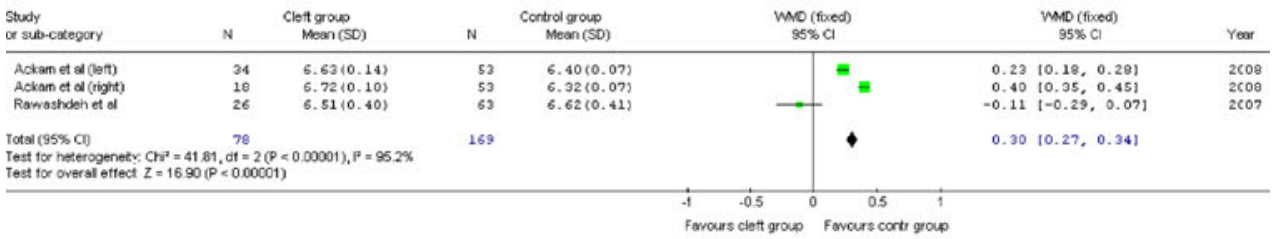

Review: MO Tocth Size Clen:
Comparison: madisy 1 st nolex

olecomen clen site ve cortid

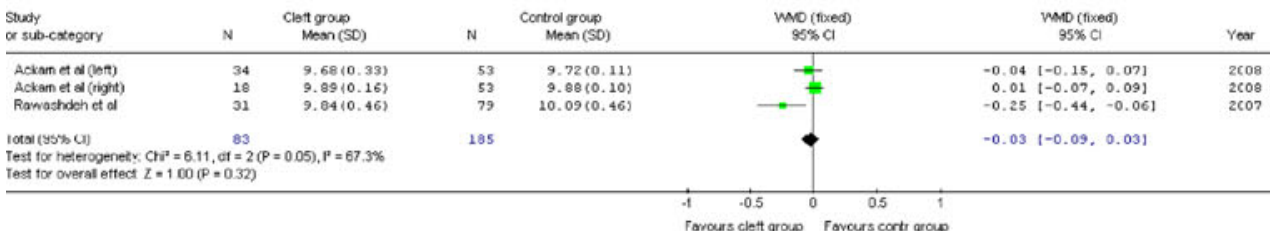

Review: Mo Tocth size clen

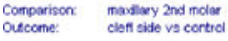

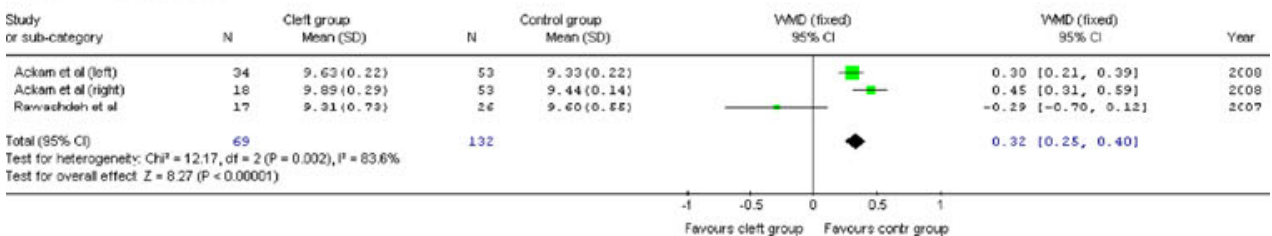


Fig. 6 Forest plots representing MD tooth dimensions for the maxillary arch on the non-cleft side in unilateral CLP patients and the same side in control patients. The studies are listed in chronological order and refer to the studies summarized in Table 1 . Shown for every study is the sample size $(N)$, weighted mean difference (WMD) between the cleft and non-cleft side, as well as the $95 \%$ confidence interval $(95 \% \mathrm{CI})$ for each measurement. The diamonds represent the overall WMD and $95 \%$ CI. $I^{2}$ values and $\chi^{2}\left(\mathrm{Chi}^{2}\right)$ values (for heterogeneity) and $p$ values (for statistical significance) are shown below each forest plot

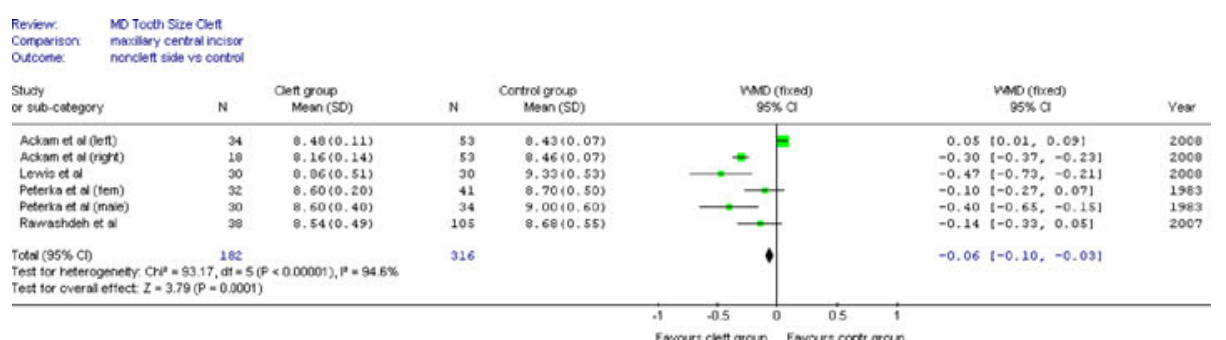

Rownew. Mo Tocth Size Clett
Comperison maxiluy hteral inciso

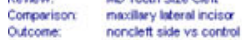

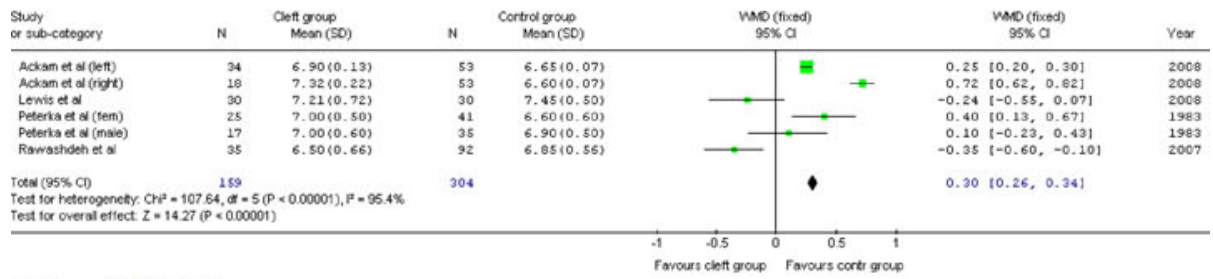

Renew. Mo Tocth Sire Clett

Compoxison maxilsy carime

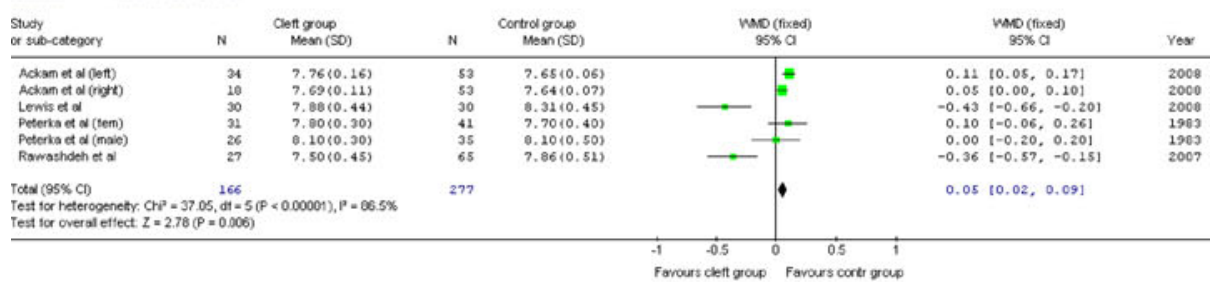

Review Mo Tocth Size clett

Comporison maxilisy stst premole

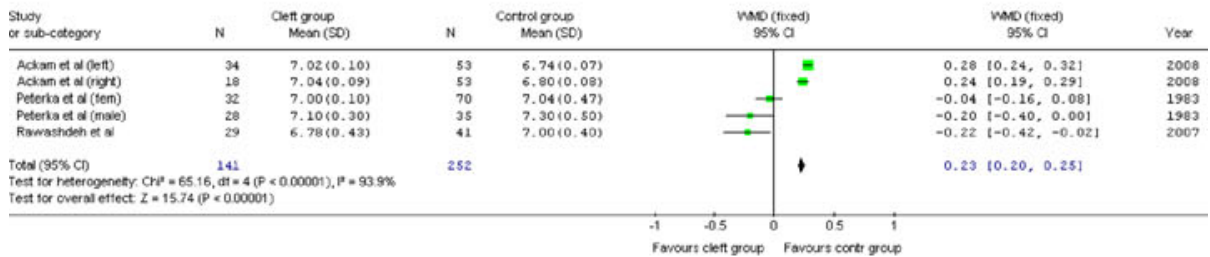

Review. Mo Touth Size Clett
Comperison maxiley zad premoly.

atconen nerclet sde ve cort

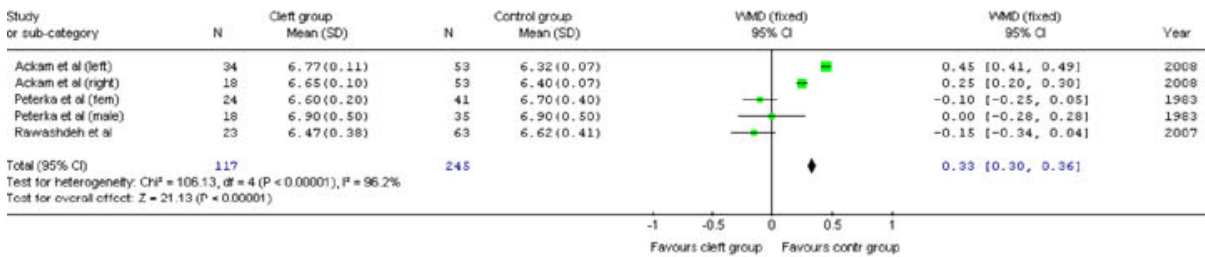

Review Mo Tocth Size Clett
Comperison maxilyy 1 it molor

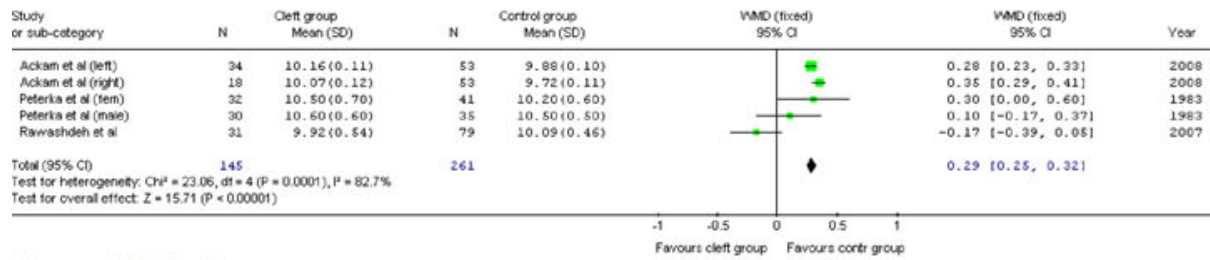

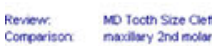

Comperison maxiliey y and molar

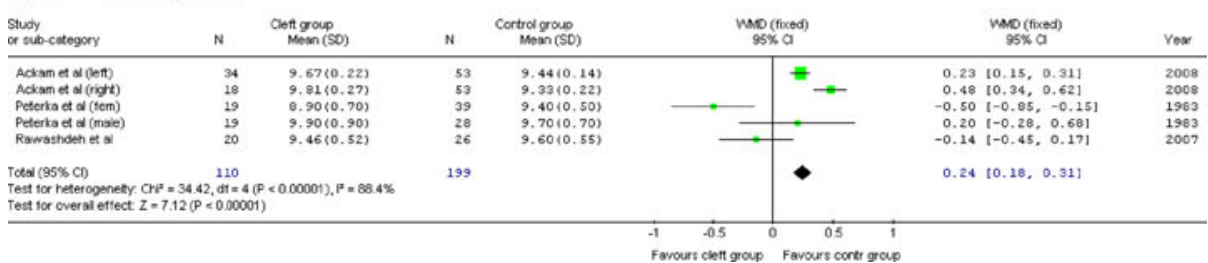


Fig. 7 Forest plots representing MD tooth dimensions for the mandibular arch on the noncleft side in unilateral CLP patients and the same side in control patients. The studies are listed in chronological order and refer to the studies summarized in Table 1. Shown for every study is the sample size $(N)$, weighted mean difference (WMD) between the cleft and non-cleft side, as well as the $95 \%$ confidence interval $(95 \%$ CI) for each measurement. The diamonds represent the overall WMD and $95 \%$ CI. $I^{2}$ values and $\chi^{2}\left(\mathrm{Chi}^{2}\right)$ values (for heterogeneity) and $p$ values (for statistical significance) are shown below each forest plot

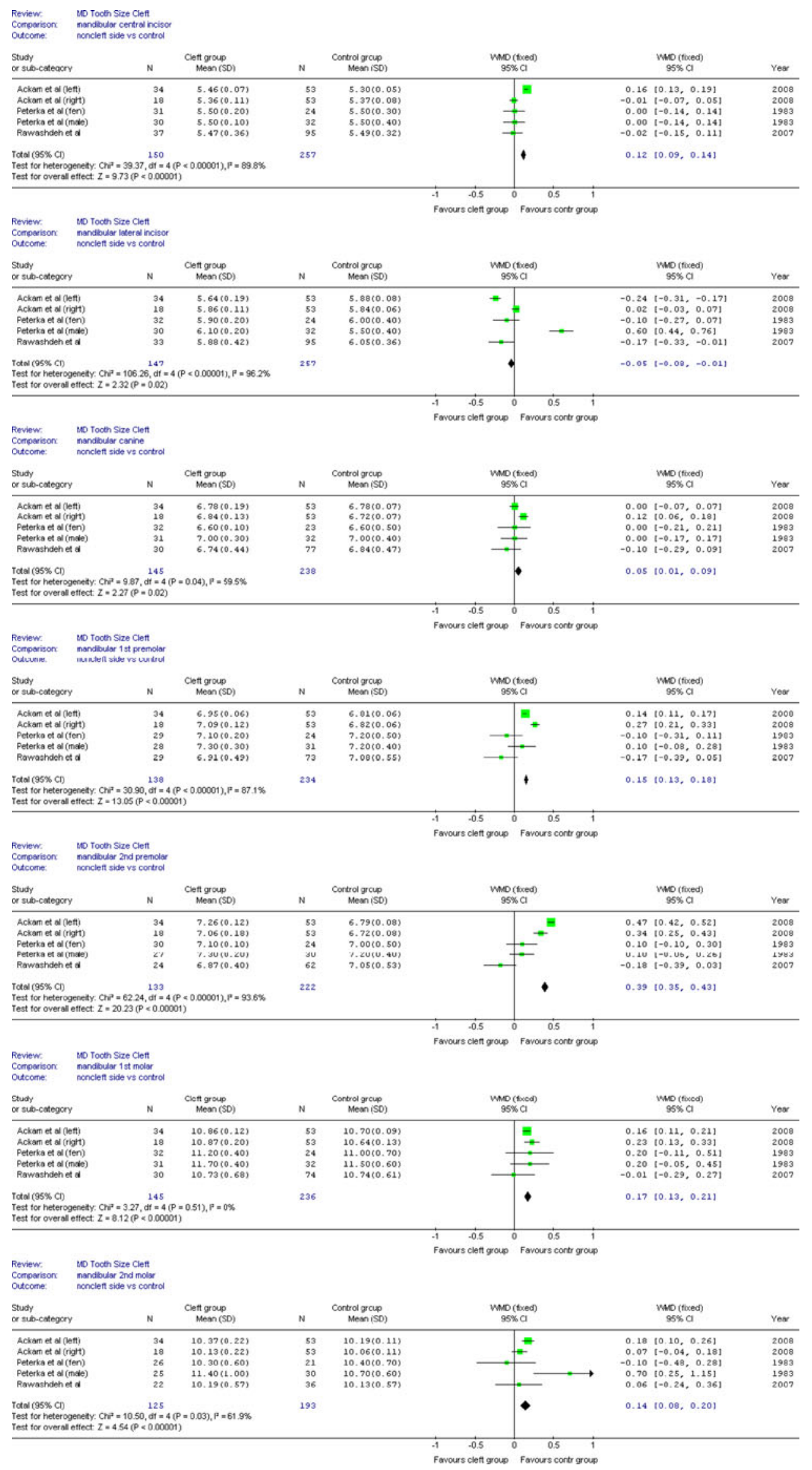




\section{Discussion}

Using meta-analyses methodology, which consists of a clearly formulated question and explicit methods to identify, select, and analyze relevant research [34], the present study demonstrates that non-syndromic unilateral CLP patients tend to have larger posterior teeth both on the cleft and the non-cleft side, when compared with the general population. This is an unexpected finding as it seems to contradict the widely held belief that CLP patients have smaller teeth than control subjects. Most articles in the literature report a tooth size reduction in CLP patients $[1,7,11,14,16,17,19,20$, 35], although they tend to generalize results. This inconsistency of the present finding with the results of other studies may be due to the bias arising from small sample sizes of individual studies as Lewis et al. [16] have reported and an overgeneralization when it comes to interpretation of results.

Despite the general reports that teeth in CLP patients are smaller than in controls, some studies maintain that certain teeth in CLP patients are larger than in the general population, while others find no difference. Akcam et al. [1], for example, state that maxillary and mandibular premolar MD dimensions are larger in CLP than in control patients, which is in agreement with the present results. Peterka and Mullerova [33], on the other hand, report that there are no remarkable differences in the MD widths between individuals with CPL and a control group.

Although, on the whole, teeth of non-syndromic unilateral CLP patients tend to be larger than those of the general population, the present study also found that the cleft side central and lateral maxillary incisors are smaller mesiodistally than in the general population. The canines, however, noted for their developmental stability [36], do not show important differences. The finding concerning the maxillary incisors was expected and consistent with previous findings in the literature showing the central and lateral maxillary incisors to be the most affected teeth in the cleft region $[1,7$, 14, 16, 17, 19, 35]. Maxillary lateral incisors with abnormal morphology have been reported in up to $94 \%$ of patients with cleft lip with or without cleft palate, on the cleft side [14]. Abnormal morphology of the maxillary central incisor on the cleft side has also been reported [37]. The significant size reduction in the maxillary central and lateral incisors on the cleft side of non-syndromic unilateral CLP patients could be explained on the basis that both are emerging from the medial nasal process that is claimed to be deficient [11]. In addition, direct damage during surgical repair on the permanent teeth cannot be ruled out $[13,38]$.

As far as the comparison between the cleft and non-cleft side is concerned, the results of the present study reveal that non-syndromic unilateral CLP patients show a trend towards smaller maxillary but larger mandibular teeth on the cleft than the non-cleft side. More specifically, it was found that central incisors, lateral incisors, and first molars in the maxilla are smaller in the cleft than in the non-cleft region. Previous studies have also cited that maxillary central and lateral incisors are smaller on the cleft side [11, 16, 19]. Sofaer [23] and Werner and Harris [7] report high levels of tooth size asymmetry between the cleft and non-cleft sides. In the mandible, analysis of the present data shows that incisors and premolars are larger on the cleft side, while the other teeth do not demonstrate any differences. Rawashdeh and Bakir [11] similarly found that in the mandibular arch, lateral incisor, and premolars were larger on the cleft than the non-cleft side.

An indisputable explanation as to why there are differences between tooth sizes in CLP patients and the general population has yet to be published. Several authors have suggested various propositions. Some claim that an underlying genetic link may exist between clefting and tooth size, while others maintain that there is likely to be a direct local effect on the developing tooth germs where the cleft involves the alveolus, rather than a genetic effect [19]. A hypothesis states that the underlying embryonic disturbance which leads to clefts is widely spread and can affect other tissues and organs of the body in various unpredictable ways. It may thus be expected that the influence on various units of the dentition, which are in various phases of morphogenesis and morphodifferentiation throughout the late embryonic and fetal periods, may be reflected by variations in tooth size and morphology [38]. Morphological and size irregularities of the dental crowns in individuals affected with CLP occur throughout the entire dentition and not merely in the maxillary units in the immediate area of the cleft [39]. The mandibular dentition is also affected. Whereas the most extreme dental abnormalities are almost always limited to the vicinity of the cleft, less severe abnormalities either appear in other regions or are generalized throughout the dentition [23]. If clefting was an isolated phenomenon incurred during early fetal development, the permanent teeth that form much later (especially those teeth away from the cleft site and in the mandible) should be unaffected. If, on the other hand, clefting is simply one of the more overt sequelae of a compromised growth potential, it would follow that the whole dentition would in some way be affected [7].

The results suggest that isolated CLP is not just a localized transient disruption in development. The systemic compromised growth potential is expressed in these data as alterations in tooth size and amplified asymmetry in both dental arches. It cannot be ruled out however that the cleft itself, which interferes with feeding, respiration, and many other physical and psychological functions, is not in part responsible for the observed alterations in tooth size [7].

Abnormally high levels of tooth-size asymmetry have been found in children with oral clefts. The asymmetry may be to 
some extent under genetic control [23]. Asymmetric development of contralateral teeth in both jaws is far more common in these children. This asymmetry has been suggested to be a milder expression of hypodontia [14]. Increased fluctuating dental asymmetry is proposed to be an indication of amplified developmental instability, such as that occurring in CLP [40]. Considering all tooth types, maxillary teeth are not inherently more asymmetric than their mandibular counterparts, even though the cleft is limited to the maxillary arch [7].

The actual differences in MD tooth size between CLP patients and the general population, although statistically significant, was on average less than $0.5 \mathrm{~mm}$. Where measurement errors and reliability were evaluated by duplicate measurements, both systematic error and random error were found to be very low in each of the individual studies. A reduction in tooth size of less than $0.5 \mathrm{~mm}$ however was considered clinically insignificant. The only clinical significant differences seen are for the maxillary lateral incisor (cleft vs. non-cleft; cleft side vs. control) and the mandibular second premolars (cleft side vs. control).

Individual tooth dimensions are important in the clinical assessment of proportions and ratios [16]. Orthodontists aim to achieve a stable, functional, and esthetic occlusion. In order to accomplish this, the dentition has to be in proportion; this is important not only from an aesthetic standpoint but also occlusally [41]. If teeth in CLP patients were universally smaller or larger in a systematic way, then no serious complications would arise because, aesthetically, the teeth would all look in proportion and, occlusally, would articulate properly [16]. However, according to the results of this study, the degree of difference between tooth dimensions in CLP patients and in the general population varies according to tooth type.

The present meta-analysis, following the application of the inclusion criteria, only located four suitable articles. This is unfortunately a rather low number and may suggest that results should be taken with caution. Ideally, a meta-analysis with a larger number of good quality studies would be able to provide more reliable results. However, given the state of the existing literature, the present results accurately represent the data that is currently available. The quality analysis used in the present study identified three medium-quality studies and one low-quality study. Certain limitations are however present when judging quality. Firstly, the quality assessment method used was designed for randomized clinical trials while the present study does not require a randomized clinical trial design but a case-control design, which is adequate to investigate the hypothesis. Furthermore, there are limitations in carrying out blinded measurements when comparing clefts and controls because the cleft case will mostly always be recognizable. These limitations must be taken into consideration when judging the quality of the included studies.

Further research into tooth size in cleft patients is necessary as many aspects of this still remain unclear. This should involve determination of tooth sizes in bilateral and other types of cleft cases. Different tooth size patterns may be observed in these patients assuming that the bilateral condition reflects less developmental control on average than in the unilateral [7]. Other tooth size parameters should also be investigated, apart from MD tooth dimensions, which are equally as important for optimal occlusal relationships. Finally, looking into tooth sizes of the deciduous dentition would also be valuable in order to help in the understanding of clefting and tooth size differences.

\section{Conclusions}

Non-syndromic unilateral CLP patients tend to have larger posterior teeth both on the cleft side and on the non-cleft side, when compared with the general population. Central and lateral maxillary incisors of the cleft side, however, are smaller in non-syndromic unilateral CLP patients than in the general population. The non-cleft side maxillary central and mandibular lateral incisors are also smaller in non-syndromic unilateral CLP patients than in the general population. Non-syndromic unilateral CLP patients tend to have smaller maxillary teeth on the cleft than the non-cleft side but larger mandibular teeth.

Conflicts of interest The authors declare that they have no conflicts of interest.

\section{References}

1. Akcam MO, Toygar TU, Ozer L, Ozdemir B (2008) Evaluation of 3-dimensional tooth crown size in cleft lip and palate patients. Am J Orthod Dentofacial Orthop 134:85-92

2. Bailit HL (1975) Dental variation among populations. An anthropologic view. Dent Clin North Am 19:125-139

3. Horowitz SL, Osborne RH, DeGeorge FV (1958) Caries experience in twins. Science 128:300-301

4. Osborne RH, Horowitz SL, DeGeorge FV (1958) Genetic variation in tooth dimensions: a twin study of the permanent anterior teeth. Am J Hum Genet 10:350-356

5. Araujo E, Souki M (2003) Bolton anterior tooth size discrepancies among different malocclusion groups. Angle Orthod 73:307-313

6. Lundström A (1948) Tooth size and occlusion in twins. Thesis (Uppsala) Karger: Basle

7. Werner SP, Harris EF (1989) Odontometrics of the permanent teeth in cleft lip and palate: systemic size reduction and amplified asymmetry. Cleft Palate J 26:36-41

8. Kabban M, Fearne J, Jovanovski V, Zou L (2001) Tooth size and morphology in twins. Int J Paediatr Dent 11:333-339

9. Bailit HL, Sung B (1968) Maternal effects on the developing dentition. Arch Oral Biol 13:155-161

10. Garn SM, Osborne RH, McCabe KD (1979) The effect of prenatal factors on crown dimensions. Am J Phys Anthropol 51:665-678

11. Rawashdeh MA, Bakir IF (2007) The crown size and sexual dimorphism of permanent teeth in Jordanian cleft lip and palate patients. Cleft Palate Craniofac J 44:155-162 
12. Pruzansky S (1955) The multidisciplinary approach to the treatment of cleft palate in children; integration of multiprofessional resources through a program of basic research. J Int Coll Surg 24:370-379

13. Dixon DA (1968) Defects of structure and formation of the teeth in persons with cleft palate and the effect of reparative surgery on the dental tissues. Oral Surg Oral Med Oral Pathol 25:435-446

14. Ranta R (1986) A review of tooth formation in children with cleft lip/palate. Am J Orthod Dentofacial Orthop 90:11-18

15. McCance A, Roberts-Harry D, Sherriff M, Mars M, Houston WJ (1993) Sri Lankan cleft lip and palate study model analysis: clefts of the secondary palate. Cleft Palate Craniofac J 30:227-230

16. Lewis BR, Stern MR, Willmot DR (2008) Maxillary anterior tooth size and arch dimensions in unilateral cleft lip and palate. Cleft Palate Craniofac J 45:639-646

17. Lai MC, King NM, Wong HM (2009) Abnormalities of maxillary anterior teeth in Chinese children with cleft lip and palate. Cleft Palate Craniofac J 46:58-64

18. Rawashdeh MA, Abu Sirdaneh EO (2009) Crown morphological abnormalities in the permanent dentition of patients with cleft lip and palate. J Craniofac Surg 20:465-470

19. Walker SC, Mattick CR, Hobson RS, Steen IN (2009) Abnormal tooth size and morphology in subjects with cleft lip and/or palate in the north of England. Eur J Orthod 31:68-75

20. Akcam MO, Evirgen S, Uslu O, Memikoğlu UT (2010) Dental anomalies in individuals with cleft lip and/or palate. Eur J Orthod 32:207-213

21. Camporesi M, Baccetti T, Marinelli A, Defraia E, Framchi L (2010) Maxillary dental anomalies in children with cleft lip and palate: a controlled study. Int J Paediatr Dent 20:442-450

22. Tereza GP, Carrara CF, Costa B (2010) Tooth abnormalities of number and position in the permanent dentition of patients with complete bilateral cleft lip and palate. Cleft Palate Craniofac J $47: 247-252$

23. Sofaer JA (1979) Human tooth-size asymmetry in cleft lip with or without cleft palate. Arch Oral Biol 24:141-146

24. Bishara SE, Sierk DL, Huang KS (1979) Longitudinal changes in the dento-facial relationships of unilateral cleft lip and palate subjects. Cleft Palate J 16:391-401

25. Garn SM, Smith BH, Moyers RE (1981) Structured (patterned) dimensional and developmental dental asymmetry. Proc Finn Dent Soc 77:33-36

26. Boehn A (1963) Dental anomalies in harelip and cleft palate. Acta Odontol Scand 21(Suppl 38):1-109
27. Liberati A, Altman DG, Tetzlaff J, Mulrow C, Gøtzche PC, Ioannidis JP, Clarke M, Devereaux PJ, Kleijnen J, Moher D (2009) The PRISMA statement for reporting systematic reviews and metaanalyses of studies that evaluate healthcare interventions: explanation and elaboration. BMJ 339:b2700

28. Jadad AR, Moore RA, Carroll D, Jenkinson C, Reynolds DJ, Gavaghan DJ, McQuay HJ (1996) Assessing the quality of reports of randomized clinical trials: is blinding necessary? Control Clin Trials 17:1-12

29. Petrén S, Bondemark L, Söderfeldt B (2003) A systematic review concerning early orthodontic treatment of unilateral posterior crossbite. Angle Orthod 73:588-596

30. Higgins JPT, Green S eds (2005) Cochrane handbook for systematic reviews of interventions 4.2.5 (updated May 2005). In: The Cochrane Library, Issue 3. John Wiley \& Sons, Chichester, UK

31. Higgins JPT, Thompson SG (2002) Quantifying heterogeneity in a meta-analysis. Stat Med 21:1539-1558

32. Higgins JPT, Thompson SG, Deeks JJ, Altman DG (2003) Measuring inconsistency in meta-analysis. BMJ 327:557-560

33. Peterka M, Müllerová Z (1983) Tooth size in children with cleft lip and palate. Cleft Palate J 20:307-313

34. Papageorgiou SN, Papadopoulos MA, Athanasiou AE (2011) Evaluation of methodology and quality characteristics of systematic reviews in orthodontics. Orthod Craniofac Res 14:116-137

35. Blanco R, Fuchslocher G, Bruce L (1989) Variations in arch and tooth size in the upper jaw of cleft palate patients. Odontol Chil $37: 221-229$

36. Dahlberg AA (1945) The changing dentition of man. J Am Dent Asoc 32:676-690

37. Dewinter G, Quirynen M, Heidbüchel K, Verdnock G, Willems G, Carels C (2003) Dental abnormalities, bone graft quality, and periodontal conditions in patients with unilateral cleft lip and palate at different phases of orthodontic treatment. Cleft Palate Craniofac J 40:343-350

38. Lekkas C, Latief B, Ter Rabe S, Kuijpers-Jagtman A (2001) The unoperated adult cleft patient: teeth in the cleft area. Eur J Plast Surg 24:118-122

39. Kraus BS, Jordan RE, Pruzansky S (1966) Dental anomalies in the deciduous and permanent dentitions of individuals with cleft lip and palate. J Dent Res 45:1736-1746

40. Shapiro BL (1975) Amplified developmental instability in Down's syndrome. Ann Hum Genet 38:429-437

41. Bolton WA (1958) Disharmony in tooth size and its relation to the analysis and treatment of malocclusion. Angle Orthod 28:113-130 